\title{
First Biological Monitoring in the Akarçay Basin According to the Water Framework Directive: Phytoplankton and Phytobenthos
}

\author{
Nilsun DEMIR ${ }^{1}$, Tolga ÇETIN ${ }^{2}$, Caner GÖK ${ }^{2}$, Müge ŞANAL ${ }^{1}$
}

\begin{abstract}
In this study, it was aimed to investigate of phytoplankton and phytobenthos in 19 rivers and Lake Eber for the assessment of ecological quality in the Akarçay basin. A total of 63 phytoplankton species were identified. Phytoplankton was evaluated according to sensitive species and pollution indicators in rivers. Lake Eber was determined as eutrophic and sensitive area according to phytoplankton functional groups and Q Index. The presence of phytobenthic macroalgae was detected only some sampling points in rivers. Therefore diatoms were used as phytobenthos quality element. A total of 64 diatom species were identified and diatom indices were calculated. Concerning the phytobenthos assessment, IDP index ranged between 2 and 4, and ecological status changed between medium and very bad.
\end{abstract}

Key words: Water Framework Directive, ecological quality, phytoplankton, phytobenthos, diatom

\section{Introduction}

The foundation of the legislation on water quality was laid by the Water Framework Directive (WFD) 2000/60/EC (Anonymous, 2000) and related directives in the European Union. Adopting the understanding that stand-alone assessment of physical and chemical analyses which are traditionally carried out for the determination of water quality does not suffice, WFD puts the biological monitoring activities at the core of the determination of water quality. WFD offers various steps in order to execute the biological monitoring in a systematic way. Establishing a typology system which starts with the delineation of water bodies that are the smallest manageable units of water bodies in a river basin which classifies the water bodies according to abiotic criteria sets the basis for those steps (Anonymous 2003a; b). Definition of undisturbed or least impaired reference sites which need to be identified for each type of water body is a new concept in the biological monitoring activities, and it constitutes the most important step of the biological monitoring system according to the Directive (Anonymous 2003b). Water bodies and their types were identified for Turkey (Anonymous 2014a) and the activities for determination of reference sites and country-specific indices are still ongoing. A twinning project was executed for the transposition of the WFD in the field of protection and management of water resources, and "By-law on Monitoring Surface and Ground Waters" has been drafted for protecting and monitoring waters.

\footnotetext{
${ }^{1}$ Ankara University, Faculty Agriculture, Department of Fisheries and Aquaculture, Ankara, Turkey ndemir@agri.ankara.edu.tr

${ }^{2}$ T.R. Ministry of Forestry and Water Affairs, Directorate General for Water Management, Ankara, Turkey tcetin@ormansu.gov.tr
} 
Phytoplankton and phytobenthos are listed among the biological quality elements that should be monitored in water bodies according to the WFD. In order to evaluate the phytoplankton according to the WFD, indices based on biomass, abundance and composition were developed (Anonymous 2014b; Mischke et al. 2011; Padisak et al. 2006; Philips et al. 2011). There were some researches concerning the use of phytoplankton in monitoring in Turkey (Çelekli and Öztürk 2014; Çelik and Ongun 2008; Demir et al. 2014).

Adaptation of phytobenthos to the methodologies of European countries was a more challenging process compared to the phytoplankton. As a matter of fact, phytobenthos contains all phototrophic organisms from microscopic unicellular organisms to macrophytes taller than 2 meters (Anonymous 2010) and phytobenthos includes non-macrophytic components of benthic flora accordingly. Due to the lack of practical methods to use phytobenthic macroalgae in the assessment of water quality and due to the fact that phytobenthic macroalgae are used for the assessment only in three countries (Czech Republic, Austria and Germany) (Anonymous 2015), diatoms have started to be used considering that they represent an important component of phytobenthos quality element. Many studies were executed on diatom indices for the water quality assessment (Anonymous 2014c; Dell'Uomo 2004; Gomez and Licursi 2001, Kelly 1998). Certain studies were carried out for the determination of water quality by using diatom indices in Turkey (Kalyoncu et al. 2009; Solak 2011). But this is the first study for ecological assessment of Akarçay basin according to WFD aims.

Regarding the diatom studies which were carried out in Akarçay Basin, 107 epipelic diatoms were identified as a result of the sampling on a monthly basis from four points from March to December in 2008 in Akarçay River (Kıvrak and Uygun 2012). Water quality was assessed according to epipelic diatoms and aquatic macrophytes which were sampled in the Akarçay River (Kargığlu et al. 2012). The correlation between diatom indices and environmental variables were also examined (Kıvrak et al. 2012). The algal flora of Lake Akşehir was identified by Elmacı and Obalı (1998). But this lake could not be sampled because of shallowness.

Monitoring activities were started in five basins within the scope of the harmonization process of the WFD in Turkey. In this study, it is aimed to monitor the Akarçay Basin on a basin scale and to obtain findings of phytoplankton and phytobenthos to estimate the ecological quality.

\section{Method}

Akarçay Basin is a closed basin located between Central Anatolia, Aegean and Mediterranean regions $\left(30^{\circ} 02^{\prime}-31^{\circ} 51^{\prime} \mathrm{E}\right.$ and $\left.38^{\circ} 04^{\prime}-39^{\circ} 09^{\prime} \mathrm{N}\right)$. The length of the basin is approximately $130 \mathrm{~km}$ and width is $20 \mathrm{~km}$. The main source of Akarçay River is constituted by the confluence of Aççay and Aksu streams. Akarçay can be used as drinking water in upstream but the water quality declines in downstream mainly because of agricultural and other anthropogenic activities. The river discharges into Lake Eber after $80 \mathrm{~km}$ of the source and causes eutrophication of the lake (Anonymous 2013a; Kivrak and Uygun 2012; Kivrak et al. 2012). 
The precipitation area of Akarçay Basin is $7605 \mathrm{~km} 2$ and the mean annual precipitation changes between 400 and $500 \mathrm{~mm}$. The climate of the basin is continental type with a temperature of 20-25oC in summer and 0-3oC in winter (Anonymous 2013b; Kargığlu et al. 2012).

The biological monitoring of Akarçay Basin was carried out in 20 water bodies (19 rivers and Lake Eber) in April 2013. Sampling points were identified by the Directorate General for Water Management of the Ministry of Forestry and Water Affairs (Figure 1). The locations of the sampling stations are given in Table 1. Lake Akşehir was very shallow and covered by macrophytes. Thus phytoplankton could not be sampled. Also, two streams feeding the lake were found to be dry and not sampled.

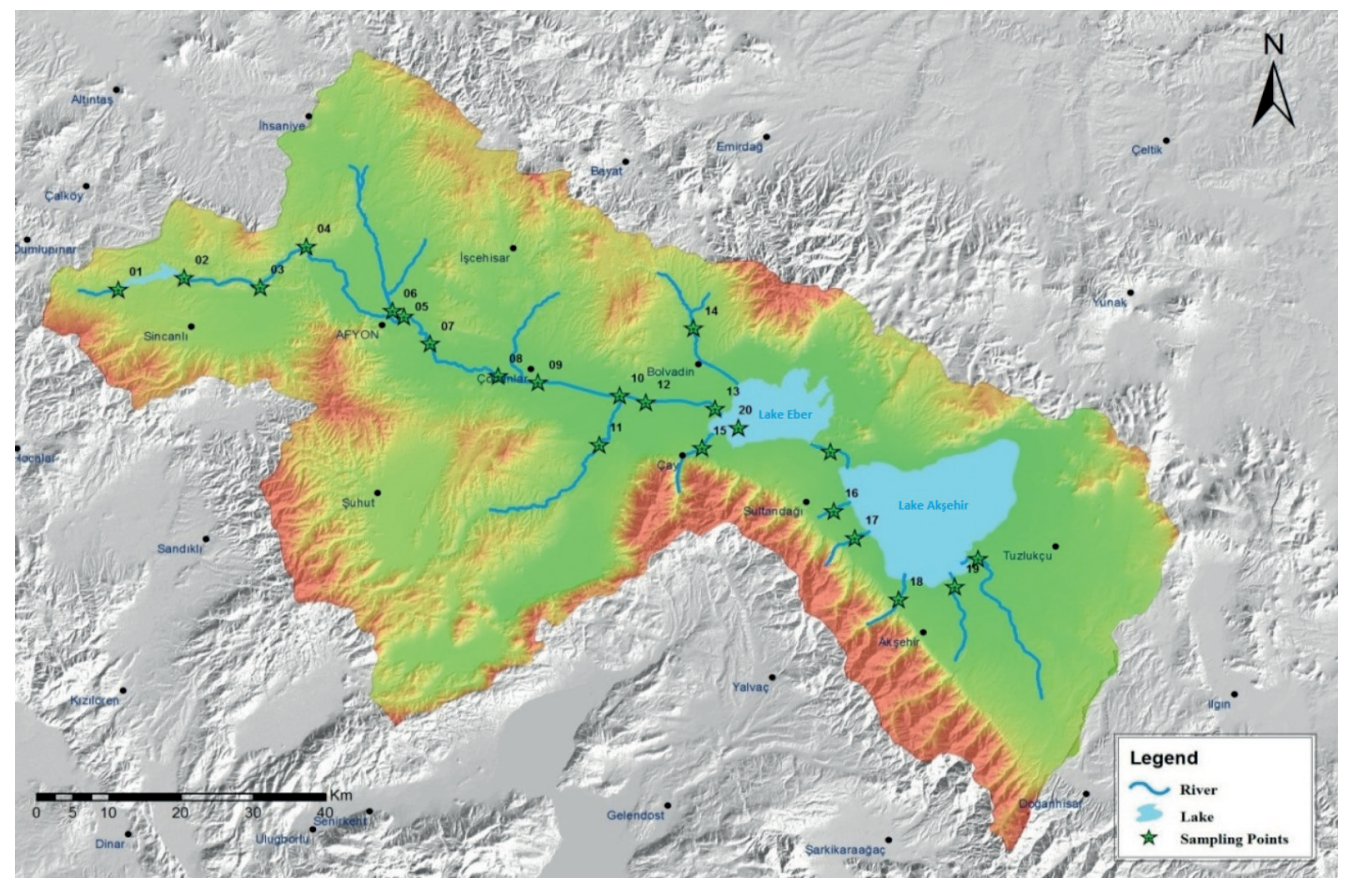

Figure 1. Map of sampling points in Akarçay Basin

Phytoplankton samples were taken from active flows in rivers and from euphotic zone (pelagial) by Ruttner sampler in Lake Eber. A plankton net ( $55 \mu \mathrm{m}$ mesh size) was used for qualitative analysis. Samples were preserved by Lugol solution. Phytoplankton were identified by a trinocular microscope (Leica DM750) and counted in 5 or $10 \mathrm{ml} \mathrm{Hydrobios} \mathrm{chambers} \mathrm{by} \mathrm{inverted} \mathrm{microscope}$ (Leica DMIL) with a camera (Anonymous 2006). The key books (John et al. 2002, Komarek and Anagnostidis 1999, 2005; Komarek and Fott 1983; Krammer and Lange-Bertalot 1985, 1986, 1988, 1991a, b; Lind and Brook 1980; Popovski and Pfiester 1990; Prescott 1973) were used for the identification. Cell dimensions of phytoplankton species were measured with a Leica DMIL microscope, digital camera and Leica Application Suite. Phytoplankton biovolume was calculated and biomass was estimated corresponding geometrical forms on the basis of wet weight (Hillebrand et al. 1999; Rott 1981). The Q Assemblage index was estimated from the biomass of phytoplankton functional 
groups in Lake Eber (Padisak et al. 2006) and ranged from 0 to 5 on a scale according to the WFD requirements. Values of the index between 0 and 1 are classified as bad, between 1 and 2 as tolerable, 2 and 3 as medium, 3 and 4 as good, and 4 and 5 as of excellent ecological quality.

Table 1.

Sampling points, codes, coordinates, water body types and locations in Akarçay Basin

\begin{tabular}{|c|c|c|c|c|}
\hline Point & Code & Coordinate & Typology & Water Body Name \& Location \\
\hline 01 & SMAKR01 & $\begin{array}{l}38^{\circ} 47^{\prime} 46.14^{\prime \prime} \mathrm{N} \\
30^{\circ} 07^{\prime} 33.48^{\prime \prime} \mathrm{E}\end{array}$ & A2R2E1Y2D1J2 & $\begin{array}{l}\text { Eğrek Stream } \\
\text { Afyon Sinanpaşa - Karacaören Village }\end{array}$ \\
\hline 02 & SMAKRY01 & $\begin{array}{l}38^{\circ} 48^{\prime} 45.05^{\prime \prime} \mathrm{N} \\
30^{\circ} 133^{\prime} 32.70^{\prime \prime} \mathrm{E}\end{array}$ & A2R2E1Y2D1J1 & $\begin{array}{l}\text { Akarçay } \\
\text { Afyon Sinanpaşa - Akdeğirmen Village }\end{array}$ \\
\hline 03 & SMAKRY02 & $\begin{array}{l}38^{\circ} 48^{\prime} 05.10^{\prime \prime} \mathrm{N} \\
30^{\circ} 20^{\prime} 45.60^{\prime \prime} \mathrm{E}\end{array}$ & A2R2E1Y2D1J1 & $\begin{array}{l}\text { Akarçay } \\
\text { Afyon Sinanpaşa - Balmahmut Village }\end{array}$ \\
\hline 04 & OMAKR03 & $\begin{array}{l}38^{\circ} 51 ' 32.96 ” \mathrm{~N} \\
30^{\circ} 24^{\prime} 47.977^{\prime \prime} \mathrm{E}\end{array}$ & A2R2E1Y2D1J1 & $\begin{array}{l}\text { Akarçay } \\
\text { Afyon - Bayramgazi Village }\end{array}$ \\
\hline 05 & OMAKR04 & $\begin{array}{l}38^{\circ} 46^{\prime} 11.60^{\prime \prime} \mathrm{N} \\
30^{\circ} 34^{\prime} 28.40^{\prime \prime} \mathrm{E}\end{array}$ & A2R2E1Y2D2J1 & $\begin{array}{l}\text { Akarçay } \\
\text { Afyon - Akçın Village }\end{array}$ \\
\hline 06 & SMAKR1102 & $\begin{array}{l}38^{\circ} 46^{\prime} 51.00^{\prime \prime} \mathrm{N} \\
30^{\circ} 33^{\prime} 27.70^{\prime \prime} \mathrm{E}\end{array}$ & A2R2E1Y2D1J2 & $\begin{array}{l}\text { Çayırbaşı Stream } \\
\text { Afyon - Organized Industrial Zone }\end{array}$ \\
\hline 07 & SMAKRY03 & $\begin{array}{l}38^{\circ} 44^{\prime} 08.40^{\prime \prime} \mathrm{N} \\
30^{\circ} 37^{\prime} 09.30^{\prime \prime} \mathrm{E}\end{array}$ & A2R2E1Y2D2J1 & $\begin{array}{l}\text { Akarçay } \\
\text { Afyon - Airport }\end{array}$ \\
\hline 08 & SMAKRY04 & $\begin{array}{l}38^{\circ} 41^{\prime} 06.82^{\prime \prime} \mathrm{N} \\
30^{\circ} 46^{\prime} 12.33^{\prime \prime} \mathrm{E}\end{array}$ & A2R2E1Y2D2J1 & $\begin{array}{l}\text { Akarçay } \\
\text { Afyon - City Center }\end{array}$ \\
\hline 09 & OMAKR0405B & $\begin{array}{l}38^{\circ} 40^{\prime} 51.74^{\prime \prime} \mathrm{N} \\
30^{\circ} 47^{\prime} 45.09^{\prime} \mathrm{E} \\
\end{array}$ & A2R2E1Y2D2J2 & $\begin{array}{l}\text { Akarçay } \\
\text { Afyon - Çobanlar }\end{array}$ \\
\hline 10 & OMAKR09 & $\begin{array}{l}38^{\circ} 40^{\prime} 09.600^{\prime \prime} \mathrm{N} \\
30^{\circ} 55^{\prime} 32.00^{\prime \prime} \mathrm{E} \\
\end{array}$ & A2R2E1Y2D2J2 & $\begin{array}{l}\text { Akarçay } \\
\text { Afyon Bolvadin - Kadıköy }\end{array}$ \\
\hline 11 & PAZARAĞAÇ & $\begin{array}{l}38^{\circ} 36^{\prime} 02.30^{\prime \prime} \mathrm{N} \\
30^{\circ} 533^{\prime} 37.64^{\prime \prime} \mathrm{E}\end{array}$ & A2R2E1Y2D2J1 & $\begin{array}{l}\text { Kali Stream } \\
\text { Afyon Çay - Maltepe Village }\end{array}$ \\
\hline 12 & OMAKR10 & $\begin{array}{l}38^{\circ} 39^{\prime} 41.46^{\prime \prime} \mathrm{N} \\
30^{\circ} 58^{\prime} 06.38^{\prime \prime} \mathrm{E}\end{array}$ & A2R2E1Y2D2J2 & $\begin{array}{l}\text { Akarçay } \\
\text { Afyon Bolvadin - Kadıköy }\end{array}$ \\
\hline 13 & OMAKR0405A & $\begin{array}{l}38^{\circ} 39^{\prime} 47.26^{\prime} \mathrm{N} \\
31^{\circ} 04^{\prime} 00.17^{\prime \prime} \mathrm{E}\end{array}$ & A2R2E1Y2D2J2 & $\begin{array}{l}\text { Akarçay } \\
\text { Afyon Bolvadin - Lake Eber Input }\end{array}$ \\
\hline 14 & OMAKR25A & $\begin{array}{l}38^{\circ} 46^{\prime} 26.00^{\prime \prime} \mathrm{N} \\
31^{\circ} 02^{\prime} 17.40^{\prime \prime} \mathrm{E} \\
\end{array}$ & A1R2E1Y1D1J2 & $\begin{array}{l}\text { Kirazlı Stream } \\
\text { Afyon Bolvadin - Dişli Village }\end{array}$ \\
\hline 15 & OMAKR27B & $\begin{array}{l}38^{\circ} 36^{\prime} 19.600^{\prime} \mathrm{N} \\
31^{\circ} 04^{\prime} 09.900^{\prime} \mathrm{E}\end{array}$ & A2R3E2Y2D1J1 & $\begin{array}{l}\text { Çay Stream } \\
\text { Afyon Bolvadin - Yeşilyurt Village }\end{array}$ \\
\hline 16 & SMAKR17 & $\begin{array}{l}38^{\circ} 30^{\prime} 12.80^{\prime \prime} \mathrm{N} \\
31^{\circ} 17^{\prime} 47.30^{\prime \prime} \mathrm{E}\end{array}$ & $*$ & Afyon Sultandağı - Dereçine Village \\
\hline 17 & SMAKR19 & $\begin{array}{l}38^{\circ} 28^{\prime} 32.80^{\prime \prime} \mathrm{N} \\
31^{\circ} 19^{\prime} 43.90^{\prime \prime} \mathrm{E}\end{array}$ & $*$ & Konya Akşehir - Gölçayır Village \\
\hline 18 & SMAKR21 & $\begin{array}{l}38^{\circ} 24^{\prime} 29.80^{\prime \prime} \mathrm{N} \\
31^{\circ} 22^{\prime} 51.40^{\prime \prime} \mathrm{E}\end{array}$ & A1R3E2Y2D1J1 & $\begin{array}{l}\text { Nazilli Stream } \\
\text { Konya Akşehir - Atakent Village }\end{array}$ \\
\hline 19 & OMAKR22 & $\begin{array}{l}38^{\circ} 26^{\prime} 19.90^{\prime \prime} \mathrm{N} \\
31^{\circ} 28^{\prime} 23.50^{\prime \prime} \mathrm{E}\end{array}$ & A2R2E1Y2D1J2 & $\begin{array}{l}\text { Adıyan Stream } \\
\text { Konya Akşehir - Karabulut Village }\end{array}$ \\
\hline 20 & SMAKL03 & $\begin{array}{l}38^{\circ} 36^{\prime} 56.10^{\prime \prime} \mathrm{N} \\
31^{\circ} 07^{\prime} 34.30^{\prime \prime} \mathrm{E}\end{array}$ & R2D1A2J1 & $\begin{array}{l}\text { Lake Eber } \\
\text { Afyon Bolvadin }\end{array}$ \\
\hline
\end{tabular}

*Typology of the water body could not be determined since the Strahler order is lower than 3. 
In the stream bed and cross sections, macroalgae was observed visually and abundance of macroalgae was estimated on a scale between 1 and 5 (Anonymous 2010). Samples were preserved by Lugol solution and dominant macroalgae were identified using John et al. (2002). Diatoms were scraped with a brush from submerged substrates primarily from removable cobbles or small boulders (epilithic). When there was no removable stone present in the stream bed, samples were collected from submerged common reeds (Phragmites australis (Cavanilles) Trinius et Steudel). P. australis is an ideal macrophyte for the sampling of diatoms (epiphytic) (King et al. 2006). Submerged parts of reeds were cut and placed in $300 \mathrm{ml}$ container with some water and shaken vigorously. Plants were removed from the container and samples were preserved (Anonymous 2004). If the bottom structure was not rocky which was composed of sand, silt, particulate organic matter or clay, sediment samples were taken by a pipe (epipelic) and preserved (Anonymous 2002).

Epiphytic diatoms were sampled from the reed belt in the middle of Lake Eber. Hydrogen peroxide was used for the cleaning of diatom frustules in lab and slides were prepared by Naphrax (Anonymous 2004). Diatoms were identified with trinocular Leica microscope-camera using relevant taxonomic literature (Cox 1996, Krammer and Lange-Bertalot 1985, 1986, 1988, 1991a, b, Lange-Bertalot 2013). Diatom indices such as SLA, DES, IDG, SHE, TDI, CEE, IPS, IDAP and IDP were calculated by using OMNIDIA 5.3 (Lecointe et al., 1993). Results were evaluated according to IDP (Gomez and Licursi 2001). IDP is classified between 0 and 0.5 as excellent, 0.5 and 1.5 as good, 1.5 and 2.0 as medium, 2.0 and 3.0 as bad and more than 3.0 as very bad ecological quality according to WFD requirements.

\section{Results}

A total of 63 phytoplankton species were identified from sampling points of Akarçay Basin, which belongs to Bacillariophyta (25), Chlorophyta (23), Ochrophyta (2), Cryptophyta (2), Cyanobacteria (8) and Euglenozoa (3) (Table 2). In rivers, phytoplankton biomass was quite low and mostly it was consisted of periphytic species which belong to Bacillariophyta division. Phytoplankton Q index could be applied only to Lake Eber among the sampling points in Akarçay Basin. Because true phytoplankton community did not occur in rivers in Akarçay Basin.

In Lake Eber, it was concluded that $\mathrm{J}$ group which was one of the dominant functional groups in small and lakes rich in nutrients accounts for $36 \%$ of total phytoplankton biomass, and $\mathrm{J}$ group was followed by $\mathrm{C}$ with $25 \%$ which is dominant in eutrophic and small-medium sized lakes. Also F (\%9.5), X1 (\%7.8) and S1 (\%5.8) groups were found. In the Akarçay Basin, Lake Eber was the only water body where phytoplankton index was used. Q index was predicted as 2.57 (medium ecologic status) based on phytoplankton functional groups. Although the species belong to Cyanobacteria were not proportionally high in the sampling period, the presence of some species pointed out that there might be sudden algal blooms in summer and autumn in the lake. For this reason, medium ecologic status was pre-assessment and monitoring studies should be continued. 
Phytobenthic macroalgae, Hydrurus foetidus (Villars) Travisan from Chrysophyceae was identified in the sampling points 16 and 18. Oscillatoria limosa (C. Agardh) Gomont from Cyanobacteria and Spirogyra sp. from Chlorophyta were found in the sampling point 5 pointed out organic pollution. Due to the fact that phytobenthic algae were rarely distributed between \%1-5, the assessment of indices were carried out by using diatoms. Diatom species identified in the Akarçay Basin are shown in the Table 3.

Table 2.

Total abundance, biomass and biomass based composition (\%) of phytoplankton species in Akarçay Basin

Species

$\begin{array}{llllllllllllllllllll}01 & 02 & 03 & 04 & 05 & 06 & 07 & 08 & 09 & 10 & 11 & 12 & 13 & 14 & 15 & 16 & 17 & 18 & 19 & 20\end{array}$

Achnanthidium minutissimum (Kützing) Czarnecki

Asterionella formosa Hassal

Aulacoseira granulata (Ehrenberg) Simonsen

Brebissonia lanceolata (C.Agardh)Mahoney\& Reimer

Caloneis amphisbaena (Bory) Cleve

Cyclotella meneghiniana Kützing

Diatoma vulgaris Bory

Encyonema minutum (Hilse) D.G.Mann

Fragilaria construens (Ehrenberg) Grunow

Gomphonema olivaceum (Hornemann) Brébisso

Melosira varians C.Agardh

Navicula cryptocephala Kützing

BA Navicula gregaria Donkin

Navicula lanceolata Ehrenberg

Navicula tripunctata (O.F.Müller) Bory

Nitzschia acicularis (Kützing) W.Smith

Nitzschia dissipata (Kützing) Rabenhorst

$\begin{array}{llll}3.8 & 24.1 & 0.3 & 1,0\end{array}$

-
-

Nitzschia palea (Kützing) W.Smith

- $\quad$ -

Nitzschia sigmoidea (Nitzsch) W.Smith

\begin{tabular}{llllclcccccccccccccc}
- & - & - & - & - & - & - & - & - & - & 2.1 & - & - & - & - & - & - & - & - & - \\
\hline & - & - & - & 21.5 & - & 14.0 & - & - & 11.0 & 2.1 & 7.0 & 5.9 & - & - & - & - & - & - & - \\
\hline
\end{tabular}

Rhoicosphenia abbreviata (C.Agardh) Lange-Bertalot

Sellaphora pupula (Kützing) Mereschkovksy

Surirella brebissonii Krammer \& Lange-Bertalot

Tryblionella hungarica (Grunow) Frenguelli

Ulnaria acus (Kützing)Aboal

$\begin{array}{cccccccccccccc}- & - & - & 4.7 & 8.6 & - & 1.6 & 6.5 & - & - & - & 6.4 & - & - \\ 1.2 & 14.8 & - & 1.0 & - & - & - & - & - & - & - & - & - & 1.3\end{array}$

$\begin{array}{cccc}1.2 & 14.8 & - & 1.0 \\ 23.2 & - & - & -\end{array}$

$\begin{array}{lllllllllll}7.1 & - & - & 2.1 & - & 11.0 & 3.3 & 19.1 & 2.2 & - & 5.6\end{array}$

\begin{tabular}{llll}
5.6 & - & - & 13.4 \\
\hline
\end{tabular}

$\begin{array}{llllllllll}2.3 & 2.9 & - & 2.1 & 2.9 & - & 1.8 & 0.9 & - & 0.9\end{array}$

$\begin{array}{lllllllllll}0.7 & 0.9 & 13.8 & 2.7 & 0.7 & 12.5 & 2.8 & 1.1 & 4.9 & 2.0 & 1.1\end{array}$

Ulnaria ulna (Nitzsch) Compére 6.9

$\begin{array}{lllcc}- & - & - & 0.7 & 0.9 \\ - & - & - & - & 6.9\end{array}$

Actinastrum hantzschii Lagerheim

Acutodesmus acuminatus (Lagerheim) P.M.Tsarenko

Chlamydomonas sp.

Crucigenia tetrapedia (Kirchner) Kuntze

Desmodesmus opoliensis (P.G.Richter) E.Hegewald

Dictyosphaerium ehrenbergianum Nägeli

Gregiochloris lacustris (Chodat) Marvan,

Komarek \& Comas

Monoraphidium arcuatum (Korshikov) Hindák

Monoraphidium contortum (Thuret) Kom.-Legn.

Monoraphidium irregulare (G.M.Smith) Kom.-Legn.

Monoraphidium komarkovae Nygaard

CH Monoraphidium minutum (Nägeli) Komárková-Legn.

Monoraphidium tortile (West \& G.S.West) Kom.-Legn

Oocystis lacustris Chodat

Pediastrum dublex Meyen

Pseudopediastrum boryanum (Turpin) E.Hegewald

Pseudodidymocystis planctonica (Korshikov)

E.Hegewald \& Deason

Scenedesmus quadricauda (Turpin) Brébisson

Schroederia setigera (Schröder) Lemmermann

Sphaerocystis schroeteri Chodat

Tetraedron caudatum (Corda) Hansgirg

Tetraedron minimum (A.Braun) Hansgirg

Tetrastrum staurogeniiaforme (Schröder) Lemm.

\begin{tabular}{cccccccccccccccccccc}
- & - & - & - & - & 19.7 & - & - & - & - & - & 14.9 & - & - & - & - & - & - & - & - \\
\hline 2.0 & 25.4 & 1.5 & 12.7 & 6.5 & - & 44.1 & - & 8.7 & 2.5 & 25.0 & - & 11.2 & - & - & - & - & - & - & - \\
\hline 37.0 & 5.6 & 0.4 & 0.5 & 6.0 & 0.9 & 2.5 & 3.7 & 6.4 & 4.4 & - & 0.9 & 0.7 & 0.8 & - & - & - & - & - & 1.3 \\
\hline- & - & 0.2 & 0.3 & 2.4 & - & - & 1.3 & - & - & - & - & - & 0.4 & - & - & - & - & - & - \\
\hline- & - & 12.2 & 0.7 & 17.0 & 2.2 & 1.4 & 2.4 & 12.5 & 14.5 & - & 8.2 & 5.6 & 24.5 & - & - & - & - & - & - \\
- & - & 32.0 & 37.5 & - & 21.8 & - & - & - & 26.7 & 59.8 & - & - & - & - & - & - & - & - & - \\
\hline- & - & - & - & - & - & - & - & - & - & 0.2 & - & 0.2 & - & - & - & - & - & - & - \\
\hline- & - & - & 0.3 & - & - & - & - & 1.0 & 1.4 & - & - & - & - & - & - & - & - & - & - \\
\hline & - & - & - & - & - & - & 0.7 & - & - & - & - & - & - & - & - & - & - & - & - \\
\hline- & - & 0.7 & 1.5 & - & 2.7 & 4.7 & 6.5 & - & 1.7 & - & - & 0.4 & 4.6 & - & - & - & - & - & - \\
\hline 1.3 & - & - & - & - & - & - & - & - & 2.5 & - & - & - & 15.9 & - & - & - & - & - & - \\
- & - & 25.8 & 25.5 & 24.9 & 11.1 & 15.4 & 21.6 & 35.8 & 25.0 & - & 51.7 & 37.3 & 9.5 & - & - & - & - & - & - \\
\hline- & - & - & - & - & - & - & - & - & - & - & - & - & - & - & - & - & - & - & 2.2 \\
\hline- & - & - & - & - & - & - & - & - & - & - & - & - & - & - & - & - & - & - & 23.2 \\
\hline- & - & - & - & - & - & - & - & - & 0.9 & - & - & - & - & - & - & - & - & - & - \\
\hline- & - & - & - & - & 2.2 & - & - & - & - & - & - & - & - & - & - & - & - & - & - \\
\hline- & - & - & - & - & - & - & - & - & - & - & - & - & - & - & - & - & - & - & 3.6 \\
\hline- & - & - & - & - & - & - & - & - & - & - & - & - & - & - & - & - & - & - & 9.1 \\
\hline- & - & - & - & - & - & - & - & - & - & - & - & - & - & - & - & - & - & - & 0.2
\end{tabular}

\begin{tabular}{ccccccccccccccccccccc} 
& - & - & 0.1 & 0.1 & - & - & - & - & - & 0.1 & - & - & - & - & - & - & - & - & - & - \\
\hline & - & - & - & 0.2 & 1.5 & 3.6 & 1.7 & 2.7 & 3.9 & 2.1 & - & 2.2 & - & - & - & - & - & - & - & 4.6 \\
\hline & - & - & - & - & - & - & - & - & - & - & - & - & - & - & - & - & - & - & - & 0.3 \\
\hline & 2.4 & - & - & - & - & 0.7 & - & - & - & 0.2 & - & 0.2 & - & - & - & - & - & - & - & 1.0 \\
\hline
\end{tabular}

\begin{tabular}{llllllllllllllllllll}
- & - & - & - & - & - & - & - & 0.3 & 0.1 & - & - & - & - & - & - & - & - & - & 0.7 \\
\hline
\end{tabular}




\begin{tabular}{|c|c|c|c|c|c|c|c|c|c|c|c|c|c|c|c|c|c|c|c|c|c|}
\hline & Species & 01 & 02 & 03 & 04 & 05 & 06 & 07 & 08 & 09 & 10 & 11 & 12 & 13 & 14 & 15 & 16 & 17 & 18 & 19 & 20 \\
\hline \multirow{2}{*}{$\mathrm{OC}$} & Dinobryon divergens O.E.Imhof & 5.4 & 11.3 & - & - & - & - & - & - & - & - & - & - & - & - & - & - & - & - & - & - \\
\hline & Mallomonas heterospina J.W.G.Lund & - & - & - & - & - & - & - & - & - & - & - & - & - & - & - & - & - & - & - & 0.1 \\
\hline \multirow[b]{2}{*}{ CR } & Cryptomonas ovata Ehrenberg & - & - & - & - & - & - & - & - & - & - & - & - & - & - & - & - & - & - & - & 2.0 \\
\hline & $\begin{array}{l}\text { Plagioselmis nannoplanctica (H.Skuja) G.Novarino, } \\
\text { I.A.N.Lucas \& S.Morrall }\end{array}$ & - & - & - & - & - & - & - & - & - & - & - & - & - & - & - & - & - & - & - & $* *$ \\
\hline \multirow{9}{*}{$\mathrm{CY}$} & Anabaenopsis elenkinii V.V.Miller & - & - & - & - & - & - & - & - & - & - & - & - & - & - & - & - & - & - & - & 2.0 \\
\hline & $\begin{array}{l}\text { Aphanocapsa incerta (Lemmermann) G.Cronberg \& } \\
\text { Komárek }\end{array}$ & - & - & $* *$ & - & - & - & - & - & - & - & - & - & - & - & - & - & - & - & - & 0.1 \\
\hline & Komvoporon sp. & - & - & - & - & - & - & - & - & - & - & - & - & - & - & - & - & - & - & - & 0.4 \\
\hline & Merismopedia glauca (Ehrenberg) Kützing & - & - & - & - & - & - & - & - & - & - & - & - & - & - & - & - & - & - & - & 9.3 \\
\hline & Oscillatoria sp. & - & - & - & - & - & - & - & - & - & - & - & - & 10.3 & - & - & - & - & - & - & - \\
\hline & $\begin{array}{l}\text { Planktothrix agardhii (Gomont) Anagnostidis \& } \\
\text { Komárek }\end{array}$ & - & - & - & 0.2 & 0.4 & - & - & - & - & - & - & - & - & - & - & - & - & - & - & 0.5 \\
\hline & Pseudanabaena limnetica (Lemmermann) Komárek & - & - & 5.7 & 0.5 & 0.5 & 0.4 & - & - & - & 0.2 & - & - & - & - & - & - & - & - & - & 4.9 \\
\hline & Spirulina corakiana Playfair & - & - & - & - & - & - & - & - & - & - & - & - & - & - & - & - & - & - & - & $* *$ \\
\hline & Euglena viridis (O.F.Müller) Ehrenberg & - & - & 13.8 & 2.2 & - & - & 1.3 & - & - & - & 3.2 & - & 24.2 & 3.2 & - & - & - & - & - & - \\
\hline \multirow[t]{4}{*}{$\mathrm{EU}$} & Lepocinclis acus (O.F.Müller) Marin \& Melkonian & - & - & - & - & - & - & - & - & - & - & - & - & 1.0 & - & - & - & - & - & - & - \\
\hline & Phacus caudatus Hübner & - & - & 1.1 & - & - & - & - & - & 1.6 & - & - & - & - & - & - & - & - & - & - & - \\
\hline & Total abundance (individual/ml) & 1216 & 195 & 1030 & 444 & 2737 & 6668 & 818 & 1246 & 1246 & 5385 & 1165 & 3904 & 1864 & 501 & $* * *$ & *wok & *owk & 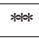 & wow & 56884 \\
\hline & Total biomass (mg wet weight/l) & 0.37 & 0.07 & 0.67 & 0.43 & 0.77 & 2.12 & 0.48 & 0.42 & 0.31 & 2.52 & 2.17 & 1.99 & 1.69 & 0.15 & wow & *wok & **ok & 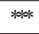 & *olk & 611 \\
\hline
\end{tabular}

\section{BA; Bacillariophyta, CH; Chlorophyta, OC; Ochrophyta CR; Cryptophyta, \\ CY; Cyanobacteria, EU; Euglenozoa}

\section{** Rarely found. *** Biomass couldn't be calculated since the phytoplankton was rarely found.}

Table 3.

The composition of phytobenthic diatom species (\%) in sampling points of Akarçay Basin

\begin{tabular}{|c|c|c|c|c|c|c|c|c|c|c|c|c|c|c|c|c|c|c|c|c|}
\hline Species & 01 & 02 & 03 & 04 & 05 & 06 & 07 & 08 & 09 & 10 & 11 & 12 & 13 & 14 & 15 & 16 & 17 & 18 & 19 & 20 \\
\hline $\begin{array}{l}\text { Achnanthidium exiguum (Grunow) } \\
\text { Czarnecki }\end{array}$ & - & - & - & - & 0.25 & - & - & - & - & - & - & - & - & - & - & - & - & - & - & - \\
\hline $\begin{array}{l}\text { Achnanthidium minutissimum (Kützing) } \\
\text { Czarnecki }\end{array}$ & 55.10 & 2.39 & 2.50 & 2.79 & 1.53 & 58.16 & 3.33 & 2.32 & 31.28 & 0.20 & 16.01 & 0.25 & 0.50 & 5.85 & 11.97 & 69.81 & 78.47 & 76.54 & 15.65 & 1.37 \\
\hline Amphora ovalis (Kützing) Kützing & - & - & - & - & - & - & - & - & - & - & - & - & - & - & - & - & - & - & 0.24 & 0.55 \\
\hline $\begin{array}{l}\text { Amphora pediculus (Kützing) Grunow } \\
\text { ex A.Schmidt }\end{array}$ & 2.35 & - & 2.27 & 3.26 & 1.53 & - & 0.67 & 0.19 & 0.47 & 0.59 & 1.72 & - & 1.25 & 0.73 & 0.70 & 0.24 & 0.50 & 0.99 & 1.47 & 10.11 \\
\hline $\begin{array}{l}\text { Brebissonia lanceolata (C.Agardh) } \\
\text { Mahoney \& Reimer }\end{array}$ & - & 0.48 & 0.68 & 0.23 & - & - & 0.22 & - & 6.64 & - & - & - & - & 0.24 & - & 0.48 & 0.25 & - & 0.24 & - \\
\hline Caloneis amphisbaena (Bory) Cleve & - & - & - & - & - & 0.95 & 0.22 & 0.19 & - & 0.20 & - & 0.50 & 3.75 & - & - & - & - & - & 0.24 & - \\
\hline Caloneis silicula (Ehrenberg) Cleve & - & - & - & - & 0.25 & - & - & - & 0.47 & - & - & - & - & - & - & - & - & - & - & - \\
\hline Cocconeis pediculus Ehrenberg & 0.20 & 0.48 & 1.36 & 0.47 & - & - & - & - & - & - & - & - & - & - & - & - & - & - & 0.24 & - \\
\hline Cocconeis placentula Ehrenberg & 0.59 & 0.48 & 3.41 & 0.23 & - & - & 0.67 & 0.39 & 1.66 & 0.40 & 1.48 & - & - & - & - & - & - & 1.23 & 1.22 & 8.74 \\
\hline Craticula accomoda (Hustedt) D.G.Mann & - & - & 0.23 & - & - & 0.24 & - & - & - & - & 3.45 & - & - & - & 24.65 & - & - & - & - & 0.55 \\
\hline $\begin{array}{l}\text { Craticula ambigua (Ehrenberg) } \\
\text { D.G.Mann }\end{array}$ & - & - & - & - & 1.53 & - & - & - & - & - & - & - & - & - & - & - & - & - & - & - \\
\hline Craticula cuspidata (Kützing) D.G.Mann & - & - & 0.68 & - & 0.51 & - & 2.66 & - & - & - & - & - & - & 0.24 & - & - & - & - & 0.49 & - \\
\hline $\begin{array}{l}\text { Cymatopleura elliptica (Brébisson) } \\
\text { W.Smith }\end{array}$ & 0.39 & - & 3.18 & 0.47 & - & - & - & - & - & - & - & - & - & - & - & - & - & - & 0.73 & - \\
\hline Cymatopleura solea (Brébisson) W.Smith & 0.59 & - & 0.45 & 0.23 & - & - & 0.22 & - & 1.42 & - & 0.99 & 0.25 & - & - & - & - & - & - & - & - \\
\hline Cymbella excisa Kützing & 4.71 & 2.63 & 1.82 & 0.70 & 0.51 & - & 1.33 & - & 1.18 & - & - & - & - & - & - & - & - & - & 0.98 & - \\
\hline $\begin{array}{l}\text { Cymbopleura naviculiformis (Auerswald } \\
\text { ex Heiberg) Krammer }\end{array}$ & - & - & - & - & - & - & - & - & 0.47 & - & - & - & - & - & 0.23 & - & - & - & - & - \\
\hline Cymbella neocistula Krammer & - & 4.77 & - & - & - & - & - & - & - & - & - & - & - & 0.98 & 4.46 & - & 0.25 & 0.74 & 0.73 & 7.10 \\
\hline Diatoma mesodon (Ehrenberg) Kützing & - & - & - & - & - & - & - & - & - & - & - & - & - & 1.22 & 1.41 & - & - & 0.49 & 0.49 & - \\
\hline Diatoma tenuis C.Agardh & - & 0.48 & 0.45 & 0.47 & 2.80 & 0.71 & 4.43 & 7.53 & 0.47 & 1.78 & - & 1.75 & 6.00 & 8.54 & - & - & - & - & - & - \\
\hline Diatoma vulgaris Bory & - & - & 2.50 & - & - & 0.47 & - & - & - & - & 6.40 & - & - & 1.22 & 2.58 & 0.48 & - & 0.25 & 8.31 & - \\
\hline Diploneis separanda Lange-Bertalot & - & - & - & - & - & - & - & - & - & 0.4 & - & - & - & - & - & - & - & - & - & - \\
\hline Encyonema minutum (Hilse) D.G.Mann & 1.18 & 0.48 & 1.59 & - & 0.51 & 2.36 & - & - & - & - & - & - & 3.00 & 1.95 & 7.28 & 0.97 & 1.24 & 0.49 & 5.62 & 3.55 \\
\hline Epithemia sorex Kützing & - & - & - & - & - & - & - & - & - & - & 1.72 & 0.75 & - & - & - & - & - & - & - & - \\
\hline $\begin{array}{l}\text { Fallacia pygmaea (Kützing) Stickle \& } \\
\text { D.G.Mann }\end{array}$ & - & - & - & - & 1.27 & - & - & 1.16 & 0.71 & 1.19 & 1.48 & - & 2.75 & 0.49 & - & 0.24 & - & - & - & 5.46 \\
\hline Fragilaria capucina Desmaziéres & 2.35 & - & 3.18 & - & - & - & - & 0.19 & - & - & - & - & - & - & - & - & - & 0.25 & 3.67 & - \\
\hline
\end{tabular}




\begin{tabular}{|c|c|c|c|c|c|c|c|c|c|c|c|c|c|c|c|c|c|c|c|c|}
\hline $\begin{array}{l}\text { Fragilaria construens } \\
\text { (Ehrenberg) Grunow }\end{array}$ & 1.96 & - & - & - & - & - & - & - & - & - & - & - & - & - & - & - & - & - & - & - \\
\hline $\begin{array}{l}\text { Fragilaria vaucheriae ( } \\
\text { Kützing) J.B.Petersen }\end{array}$ & - & - & 0.45 & - & - & - & - & 0.19 & - & - & 1.97 & 0.25 & - & 0.73 & - & - & - & 0.25 & 2.44 & - \\
\hline $\begin{array}{l}\text { Gomphonema olivaceum } \\
\text { (Hornemann) Brébisson }\end{array}$ & 2.16 & 1.91 & 1.36 & 0.47 & 1.02 & 0.47 & 0.89 & 1.35 & 0.47 & 0.59 & - & 1.00 & 0.50 & 0.73 & 2.35 & 0.24 & 0.74 & - & 1.47 & - \\
\hline $\begin{array}{l}\text { Gomphonema parvulum } \\
\text { (Kützing) Kützing }\end{array}$ & 4.51 & 1.43 & - & 1.63 & 9.16 & 3.55 & 1.77 & 7.72 & 0.47 & 5.53 & - & 4.74 & 15.00 & 6.83 & 4.93 & 0.48 & 1.49 & 1.48 & 3.67 & - \\
\hline $\begin{array}{l}\text { Gyrosigma acuminatum } \\
\text { (Kützing) Rabenhorst }\end{array}$ & 0.20 & 0.48 & 0.23 & - & - & - & - & - & 0.24 & - & - & - & - & 0.24 & - & - & - & - & 0.49 & - \\
\hline Halamphora veneta (Kützing) Levkov & - & - & - & 0.47 & 0.25 & 0.24 & - & - & - & - & - & - & - & - & - & - & - & - & - & - \\
\hline Hannaea arcus (Ehrenberg) R.M.Patrick & - & - & - & - & - & 1.89 & - & - & - & - & - & - & - & 0.49 & 9.15 & 23.43 & 5.45 & 1.98 & 9.54 & - \\
\hline $\begin{array}{l}\text { Hantzschia amphioxys } \\
\text { (Ehrenberg) Grunow }\end{array}$ & - & - & - & - & - & - & - & - & - & - & - & - & 0.50 & 0.73 & 0.23 & - & - & - & - & - \\
\hline $\begin{array}{l}\text { Hippodonta capitata (Ehrenberg) } \\
\text { Lange-Bertalot, Metzeltin \& Witkowski }\end{array}$ & - & - & - & - & 0.51 & - & - & - & - & - & - & - & 0.50 & - & - & - & - & - & - & - \\
\hline $\begin{array}{l}\text { Mayamaea atomus (Kützing) } \\
\text { Lange-Bertalot }\end{array}$ & - & - & - & 2.79 & 2.80 & - & - & - & 1.42 & - & - & - & 0.50 & 6.83 & 5.16 & - & - & 2.72 & 0.73 & 2.73 \\
\hline Melosira varians C.Agardh & - & - & 0.91 & - & 2.29 & - & - & - & 0.47 & - & - & 0.75 & - & - & - & - & - & - & 0.73 & - \\
\hline Meridion circulare (Greville) C.Agardh & - & - & 2.50 & - & - & - & - & - & - & - & - & - & - & - & - & - & - & 0.49 & 6.85 & - \\
\hline Navicula cryptocephala Kützing & 0.78 & 0.72 & - & - & 32.32 & 7.57 & - & - & 7.35 & 3.56 & 1.72 & 10.47 & 5.75 & - & 5.40 & 1.21 & 1.98 & 0.25 & 1.47 & 2.73 \\
\hline Navicula lanceolata Ehrenberg & - & 0.24 & 0.45 & - & 15.52 & 5.91 & 0.44 & - & - & 24.70 & 0.99 & 3.99 & 5.25 & 0.24 & 0.47 & - & - & 0.49 & 0.49 & 1.09 \\
\hline Navicula radiosa Kützing & - & - & - & - & - & - & - & - & - & - & - & - & - & 0.24 & - & - & - & - & - & 6.83 \\
\hline Navicula rhynchocephala Kützing & - & - & - & - & - & - & - & 0.58 & - & - & - & - & - & - & 0.23 & - & - & - & 0.73 & - \\
\hline $\begin{array}{l}\text { Navicula tripunctata } \\
\text { (O.F.Müller) Bory }\end{array}$ & 0.98 & 44.63 & 5.23 & 2.79 & 1.78 & 0.71 & 14.63 & 2.90 & 0.71 & 0.40 & 17.98 & - & 0.25 & 1.22 & 5.16 & 0,48 & 1.24 & 3.95 & 3.91 & 3.28 \\
\hline Navicula trivialis Lange-Bertalot & - & 1.19 & 1.14 & 0.70 & 1.02 & 0.95 & - & 0.19 & - & - & - & - & - & - & - & - & - & - & - & - \\
\hline Navicula veneta Kützing & 1.18 & 0.48 & 0.68 & - & - & - & - & - & - & - & - & - & - & - & - & 0,48 & 0.50 & 1.48 & 0.73 & - \\
\hline $\begin{array}{l}\text { Nitzschia acicularis } \\
\text { (Kützing) W.Smith }\end{array}$ & 0.39 & - & - & - & - & 0.47 & 3.33 & 2.32 & - & 0.59 & 1.23 & - & 0.25 & - & - & - & - & - & - & 8.74 \\
\hline Nitzschia amphibia Grunow & - & - & - & - & 2.54 & 0.24 & - & - & 0.47 & 0.20 & - & 1.25 & - & 2.20 & - & - & - & - & - & 14.21 \\
\hline $\begin{array}{l}\text { Nitzschia dissipata } \\
\text { (Kützing) Rabenhorst }\end{array}$ & 8.63 & 30.55 & 17.95 & & 3.82 & 1.18 & 18.18 & 14.29 & 17.54 & 3.95 & - & 2.74 & 8.75 & 32.68 & 8.22 & 0,72 & 3.47 & 2.47 & 10.51 & - \\
\hline Nitzschia linearis W.Smith & 2.16 & - & 2.95 & 0.70 & 0.25 & 0.24 & - & 0.19 & 0.71 & - & - & 0.25 & 0.25 & 0.24 & - & - & - & 0.25 & 4.65 & - \\
\hline Nitzschia palea (Kützing) W.Smith & - & 0.48 & 5.45 & 4.42 & 8.40 & 4.02 & 11.31 & 15.06 & 8.29 & 37.15 & 28.82 & 10.22 & 11.00 & 0.24 & - & - & 3.71 & - & - & 6.83 \\
\hline $\begin{array}{l}\text { Nitzschia sigmoidea } \\
\text { (Nitzsch) W.Smith }\end{array}$ & 0.20 & 3.58 & 7.27 & 1.16 & 0.51 & - & 0.22 & 0.19 & 1.90 & - & - & 0,50 & - & - & - & - & - & - & 0.73 & - \\
\hline $\begin{array}{l}\text { Pinnularia brebissonii } \\
\text { (Kützing) Rabenhorst }\end{array}$ & - & 0.24 & 0.68 & - & - & - & - & 0.19 & - & - & - & - & - & 0.24 & - & - & - & - & 0.24 & - \\
\hline $\begin{array}{l}\text { Planothidium lanceolatum (Brébisson } \\
\text { ex Kützing) } \\
\text { Lange-Bertalot }\end{array}$ & 0.98 & - & 0.68 & 2.79 & - & - & - & 0.19 & - & 0.40 & 1.97 & - & 2.00 & 8.78 & 3.52 & 0,48 & 0.50 & 1.73 & 1.22 & - \\
\hline $\begin{array}{l}\text { Reimeria sinuata } \\
\text { (W.Gregory) Kociolek \& Stoermer }\end{array}$ & - & - & - & - & - & - & - & - & 0.71 & - & 0.74 & - & - & - & 0.47 & 0,24 & 0.25 & 1.23 & 0.73 & - \\
\hline $\begin{array}{l}\text { Rhoicosphenia abbreviata } \\
\text { (C.Agardh) Lange-Bertalot }\end{array}$ & - & - & 0.45 & - & - & - & - & 0,19 & 0.24 & - & - & - & - & - & - & - & - & - & 0.49 & - \\
\hline $\begin{array}{l}\text { Rhopalodia gibba } \\
\text { (Ehrenberg) O.Müller }\end{array}$ & 0.20 & 0.48 & - & - & - & - & - & - & - & - & - & - & 0.25 & - & - & - & - & - & - & - \\
\hline $\begin{array}{l}\text { Sellaphora pupula } \\
\text { (Kützing) Mereschkovksy }\end{array}$ & 1.18 & - & 2.95 & 0.23 & 0.25 & 0.24 & 0.67 & 1.16 & 0.47 & - & 1.48 & 4.24 & 7.50 & 0.98 & - & - & - & - & 0.24 & 0.82 \\
\hline Surirella angusta Kützing & - & - & 0.45 & - & - & - & - & 0.19 & 0.71 & - & - & 0.25 & - & 0.24 & - & - & - & - & 0.98 & - \\
\hline $\begin{array}{l}\text { Surirella brebissonii } \\
\text { Krammer \& Lange-Bertalot }\end{array}$ & 3.92 & 0.48 & 6.59 & 0.70 & 1.53 & 1.65 & - & 2.32 & 1.66 & 10.47 & 2.46 & 4.49 & 2.25 & 0.73 & 0.47 & - & - & - & 2.44 & - \\
\hline Surirella ovalis Brébisson & - & - & - & - & - & - & - & - & - & - & - & 0.25 & - & - & - & - & - & - & - & - \\
\hline $\begin{array}{l}\text { Surirella minuta Brébisson } \\
\text { ex Kützing }\end{array}$ & 0.20 & - & - & - & - & 0.47 & - & - & - & - & 1.23 & - & - & - & 0.23 & - & - & - & - & 0.27 \\
\hline $\begin{array}{l}\text { Tryblionella hungarica } \\
\text { (Grunow) Frenguelli }\end{array}$ & 0.39 & - & 5.68 & 1.16 & 4.33 & 5.67 & 14.41 & 10.62 & 5.45 & 5.53 & 6.16 & 35.91 & 17.00 & 1.95 & - & - & - & - & 1.22 & 3.28 \\
\hline Ulnaria acus (Kützing) Aboal & - & - & - & - & - & - & - & - & 0.24 & 0.99 & - & - & - & - & - & - & - & - & 0.24 & 11.75 \\
\hline Ulnaria capitata (Ehrenberg) Compére & - & - & 0.45 & - & - & - & - & - & - & - & - & - & - & - & - & - & - & - & - & - \\
\hline Ulnaria ulna (Nitzsch) Compére & 2.55 & 0.95 & 11.14 & 3.49 & 1.02 & 1.65 & 20.40 & 28.19 & 5.45 & 1.19 & - & 15.21 & 5.25 & 11.95 & 0.70 & - & - & 0.25 & 2.69 & - \\
\hline
\end{tabular}

Results were assessed by selecting indices among the ones in the OMNIDIA programme which include diatom species identified in the Akarçay Basin (Table 4). 
Table 4.

Values of diatom indices in the sampling points of Akarçay Basin

\begin{tabular}{llllllllll}
\hline Nokta & SLA/4 & DES/5 & IDG/5 & SHE/7 & TDI/100 & CEE/10 & IPS/5 & IDAP/5 & IDP/4 \\
\hline 1 & 1.62 & 3.99 & 3.05 & 4.77 & 47.20 & 7.50 & 3.28 & 2.75 & 2.40 \\
2 & 1.28 & 4.89 & 4.03 & 4.96 & 81.90 & 7.60 & 2.59 & 3.85 & 2.11 \\
3 & 1.74 & 4.11 & 3.19 & 4.84 & 73.20 & 5.90 & 2.47 & 2.95 & 2.31 \\
4 & 1.44 & 4.65 & 3.01 & 4.67 & 94.40 & 7.10 & 1.35 & 3.06 & 2.52 \\
5 & 1.48 & 3.10 & 2.91 & 3.86 & 86.40 & 4.50 & 2.30 & 2.60 & 2.93 \\
6 & 1.71 & 3.17 & 2.88 & 4.29 & 43.50 & 7.40 & 3.69 & 2.66 & 2.87 \\
7 & 1.84 & 3.71 & 2.96 & 4.54 & 76.30 & 5.20 & 2.17 & 2.92 & 2.57 \\
8 & 1.97 & 2.85 & 2.66 & 4.31 & 74.60 & 4.20 & 1.93 & 2.63 & 2.68 \\
9 & 1.73 & 3.66 & 2.87 & 4.48 & 63.50 & 6.70 & 2.48 & 2.94 & 2.77 \\
10 & 1.52 & 2.11 & 2.73 & 3.13 & 91.10 & 3.90 & 1.85 & 2.76 & 3.21 \\
11 & 1.92 & 3.06 & 2.63 & 3.29 & 72.50 & 4.90 & 2.47 & 3.06 & 2.78 \\
12 & 2.24 & 2.41 & 2.65 & 4.17 & 77.90 & 4.10 & 2.30 & 2.37 & 2.74 \\
13 & 2.03 & 2.89 & 2.67 & 3.90 & 83.90 & 4.30 & 2.16 & 2.39 & 2.84 \\
14 & 1.53 & 4.07 & 2.99 & 4.65 & 80.30 & 6.30 & 2.04 & 2.81 & 2.31 \\
15 & 0.74 & 4.12 & 3.87 & 4.40 & 40.50 & 7.90 & 3.78 & 3.32 & 2.44 \\
16 & 0.50 & 4.04 & 4.25 & 4.39 & 20.50 & 8.90 & 4.82 & 3.11 & 2.18 \\
17 & 0.79 & 4.17 & 4.30 & 4.69 & 33.10 & 8.70 & 4.43 & 3.17 & 2.48 \\
18 & 1.33 & 4.45 & 3.42 & 4.13 & 35.70 & 8.50 & 4.36 & 3.10 & 2.09 \\
19 & 1.21 & 4.31 & 3.65 & 4.84 & 55.40 & 7.50 & 3.46 & 3.15 & 1.84 \\
\hline 0 & 1.97 & 3.22 & 2.89 & 4.71 & 82.10 & 4.90 & 2.61 & 2.95 & 2.54 \\
\hline
\end{tabular}

According to calculated index scores, the water quality of Akarçay Basin varied from medium to very bad status. It was estimated that the water quality in 18 points was bad, the quality was medium in sampling point 19 and it was very bad in sampling point 10 according to the IDP index. Index results were colored and schematized in a way to represent the water quality in 5 classes according to the WFD (Figure 2).

\section{Discussio $O$}

Phytoplankton were identified and their biomass were estimated in rivers in Akarçay Basin but indices were not been used. The most critical reason of not using indices for rivers in this study was that phytoplankton indices were developed for lakes. Furthermore phytoplankton did not occur in fast-flowing and relatively small rivers. Low phytoplankton biomass in river water bodies in Akarçay Basin also possibly resulted from the bad water quality. Phytoplankton as a biological quality element should be monitored in all categories of water bodies (rivers, lakes, transitional and coastal waters) according to the WFD (Anonymous 2000). However given the studies conducted by the EU Member States, it is known that only six member states (Belgium, Romania, Hungary, Slovakia, Germany and Lithuania) monitor phytoplankton in rivers (Anonymous 2015). The organisms in the water column change their places by drifting constantly 
towards downstream and therefore phytoplankton communities do not occur. On the other hand true phytoplankton communities could develop in slow-moving, large, lowland rivers. It was not always possible to set reliable reference conditions in these types of rivers due to the high level of natural variability. Therefore, it was not relevant to use phytoplankton as a quality element in rivers in the Akarçay Basin.

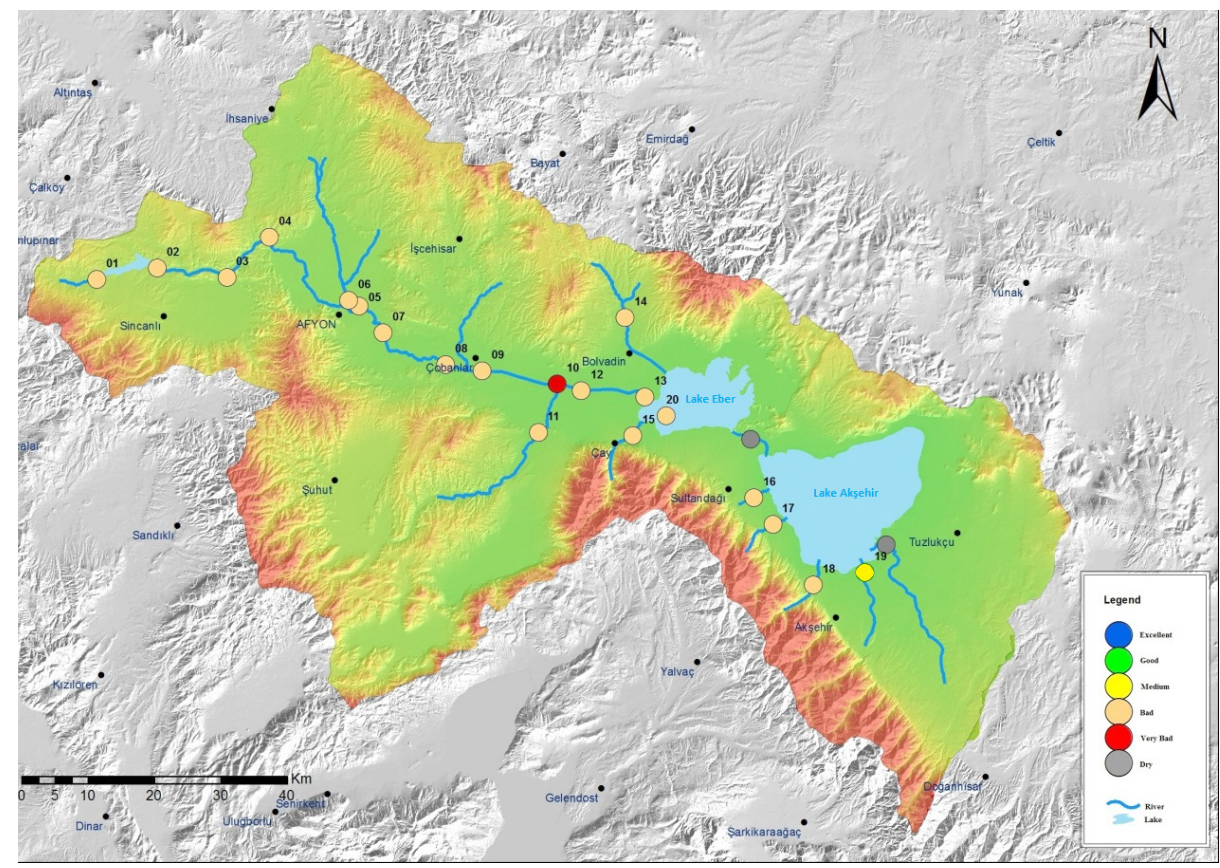

Figure 2. Ecological quality of Akarçay Basin based on phytobenthos

However it might be recommended to follow up sensitive species and/or increases in some phytoplankton species which were resistant to polisabrobic conditions such as Euglena in rivers. For instance, the fact that the biomass of the species called Euglena viridis was turned out to be as high as $24 \%$ in total phytoplankton biomass pointed out organic pollution in the sampling point 13 . The fact that the amount of suspended solid was high in rivers in the Akarçay Basin makes the counting of phytoplankton difficult. Sensitive species of phytoplankton in Turkish basins need to be defined for water quality assessments. Lake Eber was sampled as a source of stagnant surface water in Akarçay Basin. While the ecological quality was concluded as moderate according to the results of the Q index in the Lake Eber, it was identified that species/functional groups were commonly found in eutrophic waters according to Reynolds et al. (2002). The presence of functional groups reflected the conditions which were rich in nutrients and which were turbid and deficient of light conditions. Biomass of phytoplankton was estimated as $6 \mathrm{mg}$ wet weight/l. This value pointed out that the ecological quality of the lake was bad according to Sondergaard et al. (2005). Phytoplankton was sampled in April and this period was not critical in terms of phytoplankton and water quality. Therefore it is recommended to carry out monitoring activities at least in 2 periods -one in spring and the other in autumn- in Lake Akşehir and Lake Eber, and to monitor especially Cyanobacteria blooms in certain 
periods in which the water temperature rises. Concerning the phytobenthos in Lake Eber, Nitzschia amphibia can be found in various conditions from waters poor in electrolyte to waters rich in electrolyte. Ulnaria acus occurs in mesotrophic and eutrophic waters. Amphora pediculus is tolerant to $\alpha$ - $\beta$ mesosaprobic conditions (Cox 1996). Epiphytic diatom composition pointed out high organic pollution and degredation in Lake Eber. It is estimated to be a-mesosaprobic in terms of saprobity. Results of Q index indicated that Lake Eber had a moderate status, overall ecological quality turned out to be bad (poor) in the final assessment when the status was bad according to the phytobenthos. The lake could be designated as a sensitive area when eutrophication, organic pollution indicators, phytoplankton biomass and diatom indices were taken into account all together.

The water quality was estimated as moderate status according to the IDP index only in the sampling point 19 in Akarçay Basin. The water quality in the sampling point 10 was estimated as very bad status. Therefore swift action is needed. Apart from those points, the water quality was relatively better in the sampling points 16,17 and 18. The sensitive macroalgae (Hydrurus foetidus (Villars) Travisan) was identified in the sampling points 16 and 18. It has been reported that this species is found mostly in very clean water bodies or water bodies containing nutrients at a mild level (Rott et al. 2006). H. foetidus was first identified by Çevik et al. (2007) in the Şiryan River in Turkey. This species was observed in mountainous rivers in general terms and it was resistant to strong flows. Concerning the phytobenthos, Hannaea arcus -one of the diatom species- was found abundantly in the same points. It is specified that this diatom species is found mostly in very clean creeks and rivers (Lange-Bertalot 2013). The co-existence of macroalgae and diatoms stated above supports the finding that the water quality was relatively good in those points. Monitoring results of the phytobenthos indicated that the Akarçay Basin was under considerable pollution pressure. Burgan et al. (2013) reported that there was numerous pollution sources in the basin, there were no treatment plants or they did not operate in the settlements except for the city of Afyon, there were no industrial waste water treatment plants, and there were indications of fertilizers and pesticides used in the agricultural lands. Relevant ecological quality element for rivers in the Akarçay basin is diatoms. The diatom indices used in the Akarçay Basin was reflected results that were prone to pollution. Therefore diatoms should be used as phytobenthic ecological quality element in the monitoring activities to be executed in upcoming periods.

Other biological quality elements had also been studied within the scope of this study in the Akarçay Basin (Anonymous 2013c) and final ecological status had been determined based on the indicator showing the worst status. Reference values were not used in the ecological quality values in this first study which had been carried out on a basin scale and based on the biological monitoring according to the requirements of the WFD. Sampling points from which reference values can be obtained in the basin should be investigated.

\section{Acknowledgements}

This study was supported by the Directorate General for Water Management of the Ministry of Forestry and Water Affairs. We would like to thank the executives and the staff of ALKA Construction Environment Ind. Trade. Co. Ltd who had executed the project. 


\section{References}

Anonymous, (2000). Directive 2000/60/EC of the European Parliament and the council of 23 October 2000 establishing a framework for community action in the field of water policy. Official Journal of the European Communities, L327, 1-72.

Anonymous, (2002). Star Project sampling Protocol and audit benthic diatoms.

Anonymous, (2003a). Common implementation strategy for the Water Framework Directive (2000/60/EC). Guidance document no: 2. Identification of water bodies. Luxembourg, Office for official publications of the European Communities.

Anonymous, (2003b). Common implementation strategy for the Water Framework Directive (2000/60/EC). Guidance document no: 10. River and lakes - Typology, reference conditions and classification systems. Luxembourg, Office for official publications of the European Communities.

Anonymous, (2004). EN 13946. European Committee for Standardization. Water qualityGuidance standard for the routine sampling and pretreatment of benthic diatoms from rivers.

Anonymous, (2006). EN 15204. European Committee for Standardization. Water quality Guidance standard on the enumeration of phytoplankton using inverted microscopy (Uter möhl technique).

Anonymous, (2010). EN 15708. European Committee for Standardization. Water quality Guidance standard for the surveying, sampling and laboratory analysis of phytobenthos in shallow running water.

Anonymous, (2013a). Havza koruma eylem planlarının hazırlanması projesi Akarçay Havzası. Proje nihai raporu. Türkiye Bilimsel ve Teknolojik Araştırma Kurumu, Marmara Araştırma Merkezi. Gebze/Kocaeli.

Anonymous, (2013b). Yukarı Havza Sel Kontrolü Eylem Planı. Orman ve Su Işsleri Bakanlığı. Ankara.

Anonymous, (2013c). Ergene, Akarçay, Gediz, Susurluk ve Sakarya Havzalarında Su Çerçeve Direktifi Kapsamında Su Kalitesi İzleme Projesi. ALKA OSBSYGM Final Rapor No: R-AKRÇY-2013-R05, 458 s.

Anonymous, (2014a). Türkiye'de havza bazında hassas alanların ve su kalitesi hedeflerinin belirlenmesi projesi. 5. Ilerleme raporu Cilt I. Türkiye Bilimsel ve Teknolojik Araştırma Kurumu, Marmara Araştırma Merkezi. Gebze/Kocaeli. 
Anonymous, (2014b). European Commission (Joint Research Centre, Institute for Environment and Sustainability) Hoyos, C., Catalan, J., Dörflinger, G., Ferreira, J., Kemitzoglou, D., Laplace-Treyture, C., Lopez, J. P., Marchetto, A., Mihail, O., Morabito, G., Polykarpou, P., Romao, F., \& Tsiaoussi, V. Water Framework Directive intercalibration technical report, Mediterranean lake phytoplankton ecological assessment methods, Italy. EUR 26517 EN.

Anonymous, (2014c). UKTAG river assessment method. Macrophytes and phytobenthos. Phytobenthos - Diatoms for assessing river and lake ecological quality (River DAR LEQ2). Water Framework Directive - United Kingdom Technical Advisory Group.

Anonymous, (2015). (21.12.2015) Water bodies in Europe: Integrative systems to assess ecological status and recovery. http://www.wiser.eu/results/method-database/

Burgan, H. İ., İçağa, Y., Bosyanoğlu, Y. \& Kilit, M. (2013). Akarçay Akarsuyu 2006-2011 dönemi su kalite eğimi. Pamukkale Üniversitesi Mühendislik Bilimleri Dergisi, 19(3), 127-132.

Cox, E. J. (1996). Identification of freshwater diatoms from live material. Chapman \& Hall, 158 p., London.

Çelekli, A. \& Öztürk, B. (2014). Determination of ecological status and ecological preferences of phytoplankton using multivariate approach in a Mediterranean reservoir. Hydrobiologia, $740,115-135$.

Çelik, K., \& Ongun, T. (2008). Spatial and temporal dynamics of the steady-state phytoplankton assemblages in a temperate shallow hypertrophic lake (Lake Manyas, Turkey). Limnology, 9, 115-123.

Çevik, F., Whitton, B.A. \& Öztürk, O. (2007). A New Genus Record for the Freshwater Algal Flora of Turkey. Turkish Journal of Botany, 31, 149-152.

Dell'Uomo, A. (2004). L'indice diatomico di eutrofizzazione/polluzione (EPI-D) nel monitoraggio delle azque correnti, line guida. Dipartimento di Botanica ed Ecologia, Universita di Camerino.

Demir, A. N., Fakığlu, Ö. \& Dural, B. (2014). Phytoplankton functional groups provide a quality assessment method by the Q assemblage index in Lake Mogan (Turkey). Turkish Journal of Botany, 38, 169-179.

Elmacı, A. \& Obalı, O. (1998). Akşehir Gölü kıyı bölgesi alg florası. Turkish Journal of Biology, 22, 81-98. 
Gomez, N. \& Licursi, M. (2001). The Pampean Diatom Index (IDP) for assessment of rivers and streams in Argentina. Aquatic Ecology, 35, 173-181.

Hillebrand, H., Dürselen, C.D., Kirschtel, D., Pollingher U. \& Zohary T. (1999). Biovolume calculation for pelagic and benthic microalgae. J Phycol 35: 403-424.

John, D.M., Whitton, B.A. \& Brook, A.J. (2002). The freshwater algal flora of the British Isles.Cambridge, Cambridge University.

Kalyoncu, H., Çiçek, N.L., Akköz, C. \& Yorulmaz, B. (2009). Comperative performance of diatom indices in aquatic pollution assessment. African Journal of Agricultural Research, 10, 1032-1040.

Kargıı̆lu, M., Serteser, A., Kıvrak, E., İçağa, Y. \& Konuk, M. (2012). Relationships between epipelic diatoms, aquatic macrophytes, and water quality in Akarçay Stream, Afyonkarahisar, Turkey. Oceanological and Hydrobiological Studies, 41(1), 74-84.

Kelly, M.G. (1998). Use of trophic diatom index to monitor eutrophication in rivers. Wat. Res., 32 (1): 236-242.

Kivrak, E. \& Uygun, A. (2012). The structure and diversity of the epipelic diatom community in a heavily polluted stream (the Akarçay, Turkey) and their relationship with environmental variables, Journal of Freshwater Ecology, 27(3), 443-457.

Kıvrak, E., Uygun, A. \& Kalyoncu, H. (2012). Akarçay’ın (Afyonkarahisar, Türkiye) su kalitesini değerlendirmek için diyatome indekslerinin kullanılması. AKÜ FEBID 12, 27-38.

King, L., Clarke, G., Bennion, H., Kelly, M. \& Yallop, M. (2006). Recommendations for sampling littoral diatoms in lakes for ecological status assessments. Journal of Applied Phycology, 18, 15-25.

Komarek, J., \& Anagnostidis, K. (1999). Cyanoprokaryota 1. Teil: Chroococcales. In: H. Ettl, G. Gartner, H. Heynig, D. Mollenhauer. (Eds), Süsswasserflora von Mitteleuropa, Spektrum Akademischer Verlag, 548 p., Heidelberg.

Komarek, J. \& Anagnostidis, K. (2005). Cyanoprokaryota 2. Teil: Oscillatoriales. In: B. Büdel, G. Gartner, L. Krienitz, M. Schagerl (Eds), Süsswasserflora von Mitteleuropa, Spektrum Akademischer Verlag, Heidelberg, 759 p. 
Komarek, J. \& Fott, B. (1983). Chlorococcales, 7. Teil. 1Halfte. In: J. Elster and W. Ohle (Eds), Das phytoplankton des süsswassers, E. Schweizerbart'sche Verlagsbuchhandlung, 1043 p., Stuttgart.

Krammer, K. \& Lange-Bertalot, H. (1985). Naviculaceae. Bibliotheca Diatomologia, Band 9. J. Cramer, 230 p., Berlin-Stuttgart.

Krammer, K. \& Lange-Bertalot, H. (1986). Bacillariophyceae. 1. Teil: Naviculaceae. Ed: Ettl, H., Gerloff, J., Heynig, H. and Mollenhauer, D. Süsswasser flora von Mitteleuropa, Band 2/1. Gustav Fischer Verlag, 876 p., Stuttgart.

Krammer, K. \& Lange-Bertalot, H. (1988). Bacillariophyceae. 2. Teil: Bacillariaceae, Epithemiaceae, Surirellaceae. Ed: Ettl, H., Gerloff, J., Heynig, H. and Mollenhauer, D. Süsswasserflora von Mitteleuropa, Band 2/2. VEB Gustav Fischer Verlag, 596 p, Jena.

Krammer, K. \& Lange-Bertalot, H. (1991a). Bacillariophyceae. 3. Teil: Centrales, Fragilariaceae, Eunotiaceae. Ed: Ettl, H., Gerloff, J., Heynig, H. and Mollenhauer, D. Süsswasserflora von Mitteleuropa, Band 2/3. Gustav Fischer Verlag, 576 p., Stuttgart, Jena.

Krammer, K. \& Lange-Bertalot, H. (1991b). Bacillariophyceae. 4. Teil: Achnanthaceae, Kritische Ergänzungen zu Navicula (Lineolatae) und Gomphonema, Gesamtliteraturverze ichnis Teil 1-4. Ed: Ettl, H., Gärtner, G., Gerloff, J., Heynig, H. And Mollenhauer, D. Süsswasserflora von Mitteleuropa, Band 2/4. Gustav Fischer Verlag, 437 p., Stuttgart, Jena.

Lange-Bertalot, H. (2013). Diatomeen im Süßwasser - Benthos von Mitteleuropa. Koeltz Sci. Books. Germany.

Lecointe, C., Coste, M., \& Prygiel, J. (1993). “Omnidia”: software for taxonomy, calculation of diatom indices and inventories management. Hydrobiologia, 269/270, 509-513.

Lind, M. E., \& Brook, A. J. (1980). A key to the Commoner Desmids of the English Lake District. Freshwater Biol. Assoc. Publ, 123, Cumbria.

Mischke, U., Carvalho, L., McDonald, C., Skjelberd, B., Solheim, A. L., Philips, G., Hoyos, C., Borics, G., Moe, J., \& Pahissa, J. (2011). WISER Deliverable D3.1-2: Report on phyto plankton bloom metric.

Padisak, J., Borics, G., Grigorszky, I., \& Soroczki-Pinter, E. (2006). Use of phytoplankton as semblages for monitoring ecological status of lakes within the Water Framework Directive: the assemblage index. Hydrobiologia, 553, 1-14. 
Philips, G., Morabito, G., Carvalho, L., Solheim, A. L., Skjelbred, B., Moe, J., Andersen, T., Mischke, U., Hoyos, C., \& Borics, G. (2011). WISER Deliverable D3.1-1: Report on lake phytoplankton composition metrics, including a common metric approach for use in intercalibration by all GIGs. Annex Draft sensitive species.

Popovski, J. \& Pfiester, L. A. (1990). Dinophyceae (Dinoflagellida), Band 6. In: H. Ettl, J. Gerloff, H. Heynig, D. Mollenhauer, (Eds). Süsswasserflora von Mitteleuropa Gustav Fisher Verlag, 243, Jena.

Prescott, G. W. (1973). Algae of the Western Great Lakes Area, 5th edition. WM. C. Brown Co. Publ, 977, Dubuque.

Reynolds, C. S., Huszar, V., Kruk, K., Naselli-Flores, L. \& Melo, S. (2002). Towards classification of the freshwater phytoplankton. Journal of Plankton Research, 24, 417-428.

Rott, E., (1981). Some results from phytoplankton counting intercalibrations. Schweiz. Z. Hydrol., 43, 34-59.

Rott, E., Cantonati M., Füreder L. \& Pfister P. (2006). Benthic algae in high altitude stream of Alps - neglected component of aquatic biota. Hydrobiologia, 562, 195-216.

Solak, C.N., (2011). The application of diatom indices in the upper Porsuk Creek Kütahya Turkey. Turkish Journal of Fisheries and Aquatic Sciences, 11, 31-36.

Sondergaard, M., Jeppesen, E., Jensen, J.P. \& Amsinck, S.L. (2005). Water Framework Directive: Ecological Classification of Danish Lakes. Journal of App. Ecology, 42, 616-629. 


\section{Extended Turkish Abstract (Genişletilmiş Türkçe Özet)}

\section{Akarçay Havzası’nda Su Çerçeve Direktifine Göre İlk Biyolojik İzleme: Fitoplankton ve Fitobentoz}

Türkiye'de, Avrupa Birliği (AB) üyelik süreci münasebetiyle Çevre Faslı kapsamında AB mevzuatında yer alan direktiflerin uyumlaştırılması için birçok proje yürütülmektedir. Su kalitesi konusunda yürütülen çalışmaların temelini Su Çerçeve Direktifi (SÇD) (2000/60/AT) ve kardeş direktifler oluşturmaktadır. SÇD’nin uyumlaştırılması çalışmalarında ise izleme faaliyetlerinin önemi oldukça büyüktür. SÇD ile birlikte su kütlelerinin sadece fiziksel ve kimyasal açıdan izlenmesi yerini biyolojik kalite unsurları (fitoplankton, fitobentoz, makroomurgasız, makrofit, balık, makroalg ve angiosperm) temeline dayanan ekolojik bazlı değerlendirmeye bırakmıştır.

Türkiye'de ilk defa Su Çerçeve Direktifi kapsamında havza bazlı izleme çalışmalarına T.C. Orman ve Su İşleri Bakanlığı Su Yönetimi Genel Müdürlüğü tarafından 5 havzada (Akarçay, Meriç-Ergene, Susurluk, Gediz ve Sakarya) başlanmıştır. Bu çalışmalar kapsamında su kütlelerinde SÇD’ye göre biyolojik, hidromorfolojik, fizikokimyasal ve kimyasal parametrelerin izlenmesi ve uygun metotların geliştirilmesi hedeflenmiştir. Akarçay Havzası; İç Anadolu, Ege ve Akdeniz bölgelerinin kesiştiği noktada yer alan ve sularını denize ulaştıramaması nedeniyle kapalı bir havzadır. Bu nedenle kıyı ve geçiş suyu kütleleri bulunmadığı için Akarçay Havzasında sadece nehir ve göl suyu kütlelerinde izleme çalışmaları yürütülmüştür. Havzanın en büyük gölü olan Akşehir Gölü'nden, gölün sığlaşması, makrofitlerle kaplanması ve botla pelajial bölgeye ulaşılamaması nedeniyle örnek alınamamıştır. SÇD’ye göre nehir ve göllerde fitoplankton, fitobentoz, makrofit, balık ve makroomurgasızlar izlenmektedir. $\mathrm{Bu}$ çalışma Akarçay Havzasında SÇD hedeflerine uygun olarak gerçekleştirilmiş ilk çalışmadır, 2013 yılı Nisan Ayında yürütülerek 19 nehir ile 1 göl (Eber Gölü) su kütlesinde fitoplankton ve fitobentoza yönelik sonuçların elde edilmesi amaçlanmıştır.

Akarçay Havzası'nda kantitatif fitoplankton örnekleri, akarsuda aktif su akışının olduğu yerlerden, Eber Gölü'nde ise öfotik bölgeden (pelajial) Ruttner aleti ile alınmıştır. Kalitatif analizler amacıyla $55 \mu \mathrm{m}$ göz açıklığında plankton kepçesi ile plankton çekimleri yapılmıştır. Laboratuar çalışmasında öncelikle plankton kepçesi ile alınan su örnekleri mikroskop yardımıyla incelenmiş ve baskın türler teşhis edilmiştir. İkinci aşamada direkt olarak alınan su örnekleri, fitoplankton yoğunluğuna göre plankton sayım çemberlerine konmuş, Lugol solüsyonu damlatılarak bir gece bekledikten sonra fitoplankton kameralı inverted mikroskop altında fotoğraflanmış, teşhis edilmiş, sayılmış ve fitoplankton bolluğu hesaplanmıştır. Fitoplankton biyokütlesi analizinde, fitoplankton türleri teşhis edildikten sonra hücrelerin boyutları ölçülmüş, hücre hacimleri hesaplanmıştır. Örnekte bulunan hücre sayısı hücre hacmiyle çarpılarak türün örnekteki toplam hacmi belirlenmiş ve biyokütle yaş ağırlık bazında tahmin edilmiştir. Eber Gölü'nde fitoplankton fonksiyonel gruplarına göre Q indeksi hesaplanmıştır. Q indeksi 0 ile 5 arasında değişmektedir ve Su Çerçeve Direktifi'ndeki değerlendirme sistemine göre 0-1 kötü, 1-2 tolere edilebilir, 2-3 orta, 3-4 iyi ve 4-5 çok iyi ekolojik kalite sınıfını temsil etmektedir. 
Fitobentoz örneklerinin toplanması amacıyla akarsu kesitinde kıyı ve dip yapısı incelenerek su içerisinde ve su yüzeyinde uygun substratlarda makroalg varlığı araştırılmıştır. Makroalgler öncelikle makroskobik, daha sonra mikroskop yardımıyla incelenmiş ve teşhis edilmişlerdir. Diyatomeler, akarsularda öncelikle küçük kaya/taşlar gibi sert substratlar üzerinden (epilitik diyatome) firçalanarak toplanmıştır. Örnekleme yapılan su kütlesinde taş bulunmadığında diyatome örnekleri yaygın olarak bulunan kamışlar üzerinden alınmıştır. Seçilen istasyonlarda dip yapısı kayalık değilse ve yumuşak taban yapısına sahipse sediment içinde bulunan diyatomeler (epipelik) ince bir boru yardımıyla alınmıştır. Eber Gölü'nde gölün orta kesiminde bulunan kamışlık alandan epifitik diyatome örnekleri alınmıştır. Laboratuvarda diyatome früstüllerinin incelenmesi amacıyla soğuk hidrojen peroksit metodu kullanılmıştır ve Naphrax kullanılarak daimi preparatlar hazırlanmıştır. Diyatomeler mikroskop ve kamera yardımı ile fotoğraflanarak taksonomik literatüre göre teşhis edilmişlerdir. Diyatome indekslerinden SLA, DES, IDG, SHE, TDI, CEE, IPS, IDAP ve IDP, OMNIDIA programı kullanılarak hesaplanmıştır. Sonuçlar IDP indeksine göre değerlendirilmiştir. Su Çerçeve Direktifi'ndeki değerlendirme sistemine göre IDP indeksi 0-0,5 arasında çok iyi, 0,5-1,5 iyi, 1,5-2,0 orta, 2,0-3,0 kötü ve 3,0< çok kötü ekolojik durumu temsil etmektedir.

Akarçay Havzasında Bacillariophyta (25), Chlorophyta (23), Ochrophyta (2), Cryptophyta (2), Cyanobacteria (8) ve Euglenozoa'dan (3) olmak üzere toplamda 63 fitoplankton türü teşhis edilmiş ve biyokütle hesaplamaları gerçekleştirilmiştir. Akarsularda fitoplankton biyokütlesi oldukça düşük çıkmış ve indeks hesaplanamamıştır. Havzada örnek alınan noktalarda gerçek akarsu planktonunun gelişmediği gözlenmiştir ve bu nedenle Akarçay Havzası akarsularında fitoplanktonun bir kalite unsuru olarak izlenmesi uygun değildir. Fitoplankton, Avrupa'da sadece geniş nehirlerde izlenmektedir. Bu yüzden akarsularda fitoplankton sadece hassas ve kirlenme göstergesi olan türler üzerinden değerlendirilmiştir. Eber Gölü'nde ise fitoplankton fitoplanktonun değerlendirilmesi için fonksiyonel gruplardan ve aynı zamanda fitoplankton biyokütlesinden faydalanılmıştır. Fonksiyonel gruplar, Q indeksi ve biyokütle değerlendirmeleri sonucunda Eber Gölü ötrofik bir özellik göstermiş ve hassas alan olarak belirlenmiştir.

Fitobentoz açısından bakıldığında, akarsularda fitobentik makroalg varlığı sadece birkaç noktada tespit edilebildiği için ekolojik kalitenin karşılaştırılabilmesi açısından fitobentozun en önemli bileşeni olan diyatomeler kullanılmıştır. Toplamda 64 diyatome türü teşhis edilerek, diyatome indeksleri kullanılmıştır. Akarsular, 2 ve 4 arasında değişen IDP indeksi değerlerine göre antropojenik ve organik kirlenme açısından orta-çok kötü arasında ekolojik durum sergilemiştir. İleride yapılacak izleme çalışmalarında nehirler için fitobentoz izlemelerinde diyatomeler kullanılmalıdır.

Su Çerçeve Direktifi gereklilikleri doğrultusunda havza bazında ve aynı zamanda biyolojik izleme temelli gerçekleştirilen bu ilk çalışmada, hesaplanan indeks değerlerinde referans değerler kullanılamamıştır. Akarçay Havzası'nda ya da aynı su kütlesi tiplerine sahip olmak şartıyla diğer havzalarda referans alabilecek noktaların araştırılması gerekmektedir. 